\title{
The First Structural Determination of a Trivalent Californium Compound with Oxygen Coordination
}

\author{
Richard E. Sykora, Zerihun Assefa, Thomas E. Albrecht-Schmitt and Richard G. Haire
}

\section{Supplementary Information}

Raman and emission spectra were obtained on samples at both room temperature and liquid temperature using an argon-ion laser (Coherent, model 306) and a doublemeter spectrometer (Jobin-Yvon Ramanor model HG.2S). The monochromator is interfaced with a personal computer; scanning and data collections are controlled by LabSpec (version 3.04) software. Signal detection was with a water-cooled photomultiplier tube (Hamamatsu R636). The $514.5 \mathrm{~nm}$ argon laser line was used for the Raman experiments with a scanning step of $0.5 \mathrm{~cm}^{-1}$ for the monochromator. Emission spectra were collected using the $457 \mathrm{~nm}$ argon laser line with a scanning step of $6 \mathrm{~cm}^{-1}$

for the monochromator. Data analyses were performed with Grams/32 software (Galactic, version 5.1). 